\title{
NUCLÉAIRE CIVIL ET BOMBE ATOMIQUE
}

\begin{abstract}
Le Groupe des Hauts-de-Seine de la Société Française d'Energie Nucléaire a organisé le 23 mars 1982, à Paris, une réunion d'information sur le thème: « Nucléaire civil et bombe atomique ». L'intérêt des communications présentées, la qualité des débats qui ont suivi nous conduisent à publier le compte rendu complet de cette importante manifestation.
\end{abstract}

\section{Introduction}

\author{
Par A. MENEZ
}

Président du Groupe des Hauts-de-Seine de la Société Française d’Energie Nucléaire

N otre réunion a pour sujet: " Nucléaire civil et bombe atomique". Nous avons retenu ce thème après avoir fait quelques constatations :

- la première, c'est que le public confond fréquemment les applicacations militaires et les applications civiles de l'énergie nucléaire, et cela d'autant plus facilement que, me semble-t-il, l'amalgame se fait au niveau du subconscient. C'est ainsi que l'explosion de la bombe atomique d'Hiroshima est fréquemment présentée comme le péché originel de l'énergie nucléaire:

- deuxième constatation : ces deux aspects sont volontiers rapprochés dans les présentations qui sont faites dans la presse et les médias en général. Est-ce afin d'accentuer le caractère spectaculaire de la question?

- la troisième constatation que l'on pourrait faire, c'est que, si l'on pose fréquemment la question des relations et des différences entre ces deux formes de l'énergie nucléaire, on s'abstient généralement de lui apporter une réponse. Notre ambition est d'essayer de l'apporter au cours du débat qui va suivre.

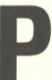
our animer ce débat, nous avons fait appel à trois éminentes personnalités:

- M. Bertrand Goldschmidt est un des pionniers français de l'énergie atomique. Né à Paris en 1912, ingénieur de l'Ecole de Physique et de Chimie, docteur ès sciences, il a été le dernier préparateur de Marie Curie qui l'avait recruté en 1933 à l'Institut du Radium, où il a travaillé jusqu'en 1940

Durant la querre, M. Goldschmidt, détaché par les Forces Françaises Libres, a participé aux recherches atomiques des alliés, en particulier sur le plutonium, aux Etats-Unis dès 1942, puis au Canada de novembre 1942 à janvier 1946, date à laquelle il dirigeait la Division de Chimie de Montréal et de Chalk River. II a été l'un des dirigeants du Commissariat à l'Energie Atomique depuis sa fondation. II fut notamment responsable de la chimie jusqu'en 1959, puis directeur des Relations internationales jusqu'en 1977. II a, à ce titre, participé aux principales négociations nucléaires où la France était présente. II a notamment représenté la France au Conseil des Gouverneurs de l'Agence Internationale de l'Energie Atomique de Vienne de 1958 à 1980 et il a présidé le Conseil des Gouverneurs en 1980.

- M. André Petit, diplômé de l'Institut d'Etudes Politiques de Paris et diplômé d'études supérieures de droit public et d'économie politique, est entré au Commissariat à l'Energie Atomique en 1950 où il a fait toute sa carrière. D'abord attaché au Secrétariat général puis chef du Service des Affaires immobilières, il est entré à la Direction des Relations internationales en 1959 comme attaché nucléaire à Bruxelles jusqu'en 1970 et, depuis lors, au siège du CEA où il occupe maintenant les fonctions de directeur adjoint des Relations internationales.
Il a plus particulièrement suivi, depuis une dizaine d'années, les questions liées à la politique de non-prolifération et aux contrôles internationaux.

- Le Contre-Amiral Louzeau est entré à l'Ecole Navale en 1947. Sa première affectation sur les sousmarins date de 1953. II a obtenu son premier commandement de sous-marin sur un sous-marin à propulsion classique, "l'Africaine », en 1958. II a suivi les cours de l'Ecole du Génie Atomique, nouvellement créée à Cherbourg, en 1959. 1960. En 1962, il a obtenu son second commandement de sousmarin, puis il est entré à. l'Ecole de Guerre. A partir de 1965, il s'est occupé du premier sous-marin nucléaire lanceur d'engins, "le Redoutable», dont il a pris le commandement en 1967, c'est-à-dire dès son lancement. II a quitté «le Redoutable » en 1972, après avoir bouclé la première patrouille csérationnelle de ce bâtiment. II a été ensuite affecté à l'Etat-Major particulier du Président de la République de 1973 à 1976. De 1976 à 1977, il a pris le commandement d'une frégate lancemissiles, "le Suffren». Ensuite, il est passé au Centre des Hautes Etudes Militaires et à l'Institut des Hautes Etudes de la Défense Nationale. Promu Contre-Amiral en 1979 il a occupé les fonctions de chef de la Division des Forces Nucléaires à l'Etat-Major des Armées. II est actuellement sous-chef d'Etat-Major "opérations " à l'Etat-Major de la Marine. 
$\mathbf{N}$ otre séance comportera une première partie comprenant les exposés des trois conférenciers. La seconde partie sera consacrée à un débat au cours duquel ils répondront aux questions.

Le premier exposé, par M. Gold- schmidt, retracera l'historique de fin, le troisième exposé, par le la prolifération. Le second exposé, Contre-Amiral Louzeau, montrera par M. Petit, évoquera l'évolution l'importance du chemin à faire pour actuelle de la prolifération, compte passer de la connaissance expéritenu des verrous technologiques, mentale aux systèmes d'armes opédes contrôles internationaux, du rationnels. traité de non-prolifération, etc. En-

A. M.

\title{
La politique de non-prolifération : historique et résultats
}

\author{
Par Bertrand GOLDSCHMIDT
}

L'auteur montre comment la politique de non-prolifération "horizontale » a été finalement un succès. D'abord, du fait qu'un nombre très restreint de pays ont accédé à l'armement nucléaire. Mais aussi parce que les efforts de non-prolifération engagés par la communauté internationale ont contribué à créer un climat politique et psycho-

\begin{abstract}
logique dissuasif à l'égard des pays tentés par la voie du nucléaire militaire. II n'y a pas de véritables verrous technologiques à la prolifération, observe l'auteur en conclusion. Ce qui compte, en définitive, c'est la volonté politique telle qu'elle s'exprime dans les Etats et dans les relations au sein de la communauté internationale.
\end{abstract}

Le 9 août 1945, le jour de la destruction de Nagasaki et de la deuxième et dernière utilisation militaire de l'arme atomique, le Président Truman a déclaré qu'il dépendait des Etats-Unis que cette arme ne soit plus jamais employée et que l'énergie nucléaire ne soit utilisée qu'à des fins pacifiques. Les Etats-Unis avaient pris le leadership de ce que nous appelons aujourd'hui la politique de non-prolifération et ils l'ont gardé pratiquement jusqu'à aujourd'hui.

A cette époque-là - et on ne s'en rendait pas compte I'humanité n'avait que très peu d'années pour revenir à un monde sans bombe, mais malheureusement elle a laissé échapper cette chance et, depuis, les dirigeants du monde se penchent sur l'angoissant problème de la prolifération des armes atomiques sous deux aspects tout à fait différents l'un, c'est la multiplication du nombre et de la puissance des armes possédées par les nations individuelles (c'est ce que l'on appelle la prolifération verticale) et l'autre, c'est la multiplication du nombre de pays possédant l'arme, c'est la prolifération horizontale. C'est surtout de celle-là dont nous allons parler aujourd'hui.

Je voudrais d'abord souligner que, dans le domaine de la prolifération verticale, l'échec a été total. Bien sûr, on ne pouvait pas prévoir en 1946 que, rapidement, il y aurait des bombes mille fois plus puissantes que celle d'Hiroshima, mais le fait est qu'aujourd'hui, essentiellement dans les arsenaux américains et soviétiques, il y a une puissance explosive nucléaire équivalente à un million de fois Hiroshima, soit environ quatre tonnes d'explosifs conventionnels par habitant du globe. C'est vous dire que les négociations sur le désarmement nucléaire sont vitales. C'est le plus grand problème politique et c'est celui qui n'a nullement été résolu.

L'autre domaine, celui de la prolifération horizontale, a été plutôt un succès. En effet, si en 1946 on avait demandé à un spécialiste de prévoir la courbe de multiplication du nombre de pays possédant l'arme, il aurait dit qu'évidemment, au fur et à mesure que les techniques se diffuseront, l'énergie nucléaire civile se développera et il y aura de plus en plus de pays qui posséderont l'arme. Eh bien, c'est justement le contraire qui est arrivé. Dans une première décennie, de 1945 à 1955, vous avez trois puissances nucléaires: les Etats-Unis en 1945, l'Union Soviétique en 1949 et le RoyaumeUni en 1952. Le Royaume-Uni, qui avait cru longtemps être le second arrivé, qui pensait arriver avant l'Union Soviétique, n'avait eu aucun scrupule, quand il avait décidé en 1947 d'avoir un programme militaire nucléaire, à être le second pays à posséder des bombes et par conséquent d'ouvrir la course à la prolifération.

\section{Heureuse surprise...}

Puis dans la décennie suivante, de 1955 à 1964, nous avons deux pays : la France en 1960 et la Chine en 1964. Et puis, dans les dix-huit ans qui se sont écoulés, depuis 1964 il n'y a eu qu'un seul pays, I'Inde, qui a fait une explosion et qui en toute probabilité n'a même pas encore commencé à mettre sur pied un armement. Enfin, pour être complet, je crois qu'il faut citer Israël, qui n'a pas procédé à une explosion, mais qui, probablement, a réuni les éléments pour avoir rapidement, ou avoir déjà, un armement atomique.

Donc, ceci est une surprise et une surprise heureuse. Je dirais que ce qui est extraordinaire et qui caractérise la discrimination de notre monde actuel entre les puissances nucléaires, celles qui possèdent l'arme et celles qui ne la possèdent pas, c'est que, d'un côté, celles qui ne la possèdent pas - et même celles qui ont des intentions douteuses - affirment le côté essentiellement pacifique de leurs recherches, tandis que les puissances nucléaires se livrent à une sorte d'exhibitionnisme, elles se vantent de la puissance de leurs armes, elles se vantent de leur efficacité, elles se vantent de leur nombre. Ceci à la fois pour avoir les sommes budgétaires considérables, peut-être en vue des négociations de limitation des armes stratégiques, et enfin essentiellement pour la dissuasion. Et c'est une chose extraordinaire de constater que ces pays sont arrivés à faire croire - et à se convaincre probablement eux-mêmes qu'ils sont les pays qui sauront le mieux ne pas utiliser leurs armes atomiques, tandis que, bien entendu, tous ceux qui lorgnent du côté du nucléaire militaire sont eux beaucoup plus dangereux pour le reste du monde. Et c'est de la même façon que l'on arrive à cette chose assez étonnante, qu'il est maintenant considéré comme contraire à l'éthique internationale pour un nouveau pays de vouloir même garder l'option ouverte sur l'armement nucléaire. Voilà donc, diraisje, l'une des réussites des efforts de non-prolifération jusqu'à maintenant que d'arriver à ce qu'un pays qui hésite à s'engager dans l'armement nucléaire ou quil garde l'option ouverte doit tenir compte de l'opinion publique mondiale. 